\title{
Stand der Technik und Entwicklung bei der berührungslosen Sortierung von Abfällen
}

\author{
State of the art and development in the field of non-contact waste sorting
}

von T. Pretz und J. Julius

\section{Kurzfassung/Summary}

Die Einzelkorntrennung anhand äußerlich identifizierbarer und berührungslos mittels geeigneter Detektoren messbarer Trennmerkmale wird als sensorgestützte Sortierung bezeichnet. Diese Technologie hat insbesondere auf dem Gebiet der trockenen Abfalltrennung die Gestaltung von Aufbereitungsverfahren revolutioniert. Dabei sind die Komplexität der technischen Ausführung und die Anzahl der eingesetzten Sensoren entscheidend für die Einsatzmöglichkeiten der Sortiermaschinen. Die fortschreitende Entwicklung neuer Sensorsysteme erschließt zudem ständig neue Anwendungsgebiete.

Single-particle separation on the basis of identifiable criteria that can be measured by suitable detectors is termed sensor-aided sorting. This is a technology that has revolutionised the design of treatment methods, especially in the field of dry waste separation systems. The main factors governing the usability of sorting machines are technological complexity and the number of sensors used. In addition, the continuing development of new sensor systems keeps opening up new fields of application.

\section{Einführung}

Die Entwicklung der Technik zum Abfallrecycling weist eine mehr als dreißigjährige Tradition auf. Die hierbei verwendeten mechanischen Aufbereitungsverfahren enthalten vor allem die Hauptgrundoperationen Zerkleinern, Klassieren und Sortieren. Während Zerkleine- rungsprozesse vorwiegend zur Reduktion der oberen Korngröße und zum Materialaufschluss dienen, wird die Klassierung zur Stoffstromteilung entsprechend unterschiedlicher Korn- oder Stückgrößen eingesetzt. Sortierprozesse sind in der Regel diesen Konditionierverfahren nachgeschaltet. Wichtige Trennmerkmale bei der Sortierung sind die Stoffdichte, die Form, die Magnetisierbarkeit, die elektrische Leitfähigkeit und diverse Oberflächeneigenschaften, wie beispielsweise die Farbe. Als Sortiertechniken wurden anfänglich praktisch ausschließlich Trennverfahren aus dem Bereich der klassischen mineralischen Aufbereitung adaptiert. Die spezifischen Eigenschaften von komplex zusammengesetzten Abfallgemischen wie geringe Schüttdichten, schlechteSchüttfähigkeiten und unterschiedlichste Kornformen erforderten jedoch eine eigenständige Entwicklung von angepassten Aufbereitungslösungen, wobei hier auch Anleihen aus verwandten $\mathrm{Ge}$ bieten, insbesondere der Nahrungsmitteltechnik, genommen wurden.

Ein entscheidender Durchbruch in der Trenntechnik für Abfälle gelang erst mit der Einführung der sensorgestützten Sortierung, die durch eine berührungslose Erkennung von Stoffeigenschaften gekennzeichnet ist. Diese Aufbereitungsmethode konnte von der rasanten Entwicklung im Bereich der Sensorik und der digitalen elektronischen Datenverarbeitung profitieren. In der ersten Phase der Anwendung derartiger Sortiermaschinen ca. Anfang 1990 wurden zunächst getrennt gesammelte Abfallgemische wie Altglas und Leichtverpackungen (LVP) aufbereitet. Dabei konnten das stoffliche Merkmal „Farbe“ mittels Transmissionsmessung bzw. die Kunststoffart mittels Spektralanalyse der Reflektion im Nahinfrarotbereich (NIR) ermittelt werden. Hinzu kam, dass die noch weit verbreitete Handsortierung durch deutlich leistungsfähigere maschinelle Systeme ersetzt wurde. So konnten die mit dem menschlichen Sehvermögen häufig nur unzulänglich identifizierbaren Eigenschaften von Abfallkomponenten durch den Einsatz sensorischer Technik erheblich genauer detektiert werden. Für eine breite Einführung der sensorgestützten Sortiertechnik waren mehrere Jahre erforderlich, in denen insbesondere die qualitativen Anforderungen an Produkte des Verpackungsrecyclings als Maßstab für die Entwicklung dienten.

Zurzeit ist eine Reihe sensorgestützter Sortiermaschinen verfügbar und in Entwicklung, die vielfach völlig „neue“ Trennmerkmale erschließen, die zuvor nicht oder nur sehr unvollkommen für eine Abfalltrennung herangezogen werden konnten. Hierbei werden erfolgreich Analyseverfahren wie die Röntgentransmissionsmessung oder die intelligente Bildverarbeitung und -auswertung adaptiert. Für die Verfahrenstechnik bedeutet dies, dass in Teilbereichen der Abfallaufbereitung, insbesondere auf dem Gebiet der trockenen Trennmethoden, Prozesse revolutioniert werden. Das Ergebnis der inzwischen fast 20-jährigen Entwicklungstätigkeit hat also $\mathrm{zu}$ einer beachtlichen Innovation bei Sortiermaschinen mit berührungsloser Detektion geführt, die das Ziel der Herstellung qualitätsgesicherter Endprodukte aus Abfällen erreichbar macht. 


\section{Grundlagen der sensor- gestützten Sortierung}

Als sensorgestützte Sortierung, auch automatische Klaubung genannt, wirddieEinzelkorntrennunganhand äußerlich identifizierbarer, berührungslos mittels Sensorik messbarer Trennmerkmale bezeichnet. Dies können u. a. die Form, die Farbe, der Glanz, die molekulare Materialzusammensetzung, die Dichte oder die elektrische Leitfähigkeit sein [Kill06]. Sortiermaschinen, die nach diesem Prinzip arbeiten, bestehen grundsätzlich aus einem Fördermittel zur Zuführung und Vereinzelung des Materials, einem Sensorsystem zur Erkennung spezifischer Materialeigenschaften einzelner Bestandteile, einersoftwaregesteuertenElektronik zur Auswertung der Signale von der Sensorik und einer mechanischen Austragsvorrichtung zur Abtrennung positiv erkannter Abfallkomponenten.

Neuentwicklungen sind heute häufig mit einer Kombination verschiedener Detektionsverfahren zur simultanen Erkennung mehrerer Materialeigenschaften ausgerüstet, deren Informationen mit Rechnern in Echtzeit auswertet werden. Diese mit Multisensorik ausgerüsteten Maschinen gewährleisten einen deutlich besseren Trennerfolg als solche mit nur einem Detektor, insbesondere für komplex zusammengesetzte Abfallgemische mit hoher Merkmalsvielfalt. Ein spezieller Vorteil moderner, sensorgestützter Sortiermaschinen ist ihre Lernfähigkeit, die auf softwaregesteuerter Datenverarbeitung basiert. Dies ermöglicht eine gute Anpassungsfähigkeit bei Änderungen der Verfahrenstechnik, der stofflichen $\mathrm{Zu}$ sammensetzung des Aufgabegutes oder der Vorgaben zur Qualität der ausgetragenen Produkte. Zudem ist bei sensorgestützten Sortiersystemen im Vergleich zu herkömmlichen Separationsverfahren die Nutzung der Sortierkriterien von dem eigentlichen mechanischen
Trennvorgang entkoppelt, so dass die Gefahr von Fehlausträgen durch gegenseitige Behinderung und Verschleppung unterschiedlicher Bestandteile verringert wird. Die Abtrennung positiv detektierter Bestandteile erfolgt bei den meisten Maschinen mittels Druckluftimpulsen aus Düsenleisten, deren Ventile von Rechnern angesteuert werden. Nur wenige Bauarten arbeiten mit mechanischen Austragsvorrichtungen wie elektromagnetisch angetriebenen Stößeln [Fea08]. Diese Maßnahme hat den Vorteil, dass eine aufwändige Drucklufterzeugung entfällt, die Reaktionsgeschwindigkeit ist jedoch geringer, so dass bei der notwendigen geringeren Fördergeschwindigkeit auch der erreichbare Durchsatz verringert wird.

Voraussetzung zur Erzielung guter Trennergebnisse mit sensorgestützten Sortierverfahren ist eine geeignete Konditionierung des Aufgabematerials. So muss in der Regel zum Erreichen einer guten Trennschärfe eine Vorklassierung durchgeführt werden, um einen möglichst engen Korngrößenbereich zu erzeugen sowie auch Fein- und Grobkorn abzutrennen, das in Abhängigkeit von der Leistungsfähigkeit und der technischen Auslegung der jeweils eingesetzten Maschinen nicht sortierfähig ist. Außerdem sollte eine vorgeschaltete Sortierung mit dem Ziel erfolgen, das zu verarbeitende Materialgemisch möglichst hoch mit dem abzutrennenden Wertstoff anzureichern und störende Bestandteile wie z. B. Kunststofffolien und andere leicht flugfähige Stoffe abzuscheiden.

Für die Güte der Erkennung und Sortierung ist letztlich entscheidend, ob es gelingt, das Aufgabematerial vor dem Durchlaufen des Messbereichs der Sensorik, beispielsweise auf einem mit ca. $3 \mathrm{~m} / \mathrm{s}$ schnelllaufenden Gurtförderer, so zu vereinzeln, dass zumindest eine Monoschicht entsteht. Dabei dürfen einzelne Komponenten nicht über- einander liegen und sollten sich gegenseitig nicht berühren. Dies wird in der Regel über eine Kaskade von Gurtförderern und/oder geneigten Schurren erreicht, die eine ansteigende Transportgeschwindigkeit gewährleisten. Mit der automatisierten Einzelkorntrennung kann Positiv- wie auch Negativsortierung durchgeführt werden. Dabei bedeutet Positivsortierung, dass Wertstoffe im ausgetragenen Produkt angereichert werden, während bei einer Negativauslese störende Bestandteile gezielt abgereichert werden.

\subsection{Maschinentechnik}

Die zur sensorgestützten Sortierung eingesetzten Geräte lassen sich entsprechend ihrer Materialzuführung im Bereich der sensorischen Erkennung in die Bauarten Rinnen- und Bandmaschinen einteilen. Die Rinnenmaschinen wurden bereits Mitte der 1980er Jahre entwickelt und zunächst praktisch ausschließlich zur Sortierung von Altglas nach Farbe bzw. Transparenz eingesetzt. Sie haben inzwischen eine sehr hohe Leistungsfähigkeit erreicht und finden insbesondere im Feinkornbereich sowie bei gut fließfähigen Schüttgütern Anwendung. Aus Abbildung 1 geht am Beispiel eines Farbsortierers der prinzipielle Aufbau des Rinnentyps hervor. Hierbei dient eine Schwingförderrinne (1) zunächst zur gleichmäßigen Verteilung des Aufgabegutgemisches über die gesamte Arbeitsbreite und zur Vorvereinzelung. Nach der Aufgabe auf eine geneigte Rinne (Schurre) wird das Material beschleunigt und weiter vereinzelt. Unterhalb der Rinne wird es dann im freien Fall von einer Erkennungseinrichtung (2) inspiziert. Diese besteht aus einer Farbzeilenkamera mit einer zugehörigen Beleuchtungseinheit. Der Zeilensensor erzeugt ein EndlosAbbild des Materialstromes, das mit einem Rechner (3) bezüglich der Farbinformation, der Position und der Größe der erkannten Bestand- 


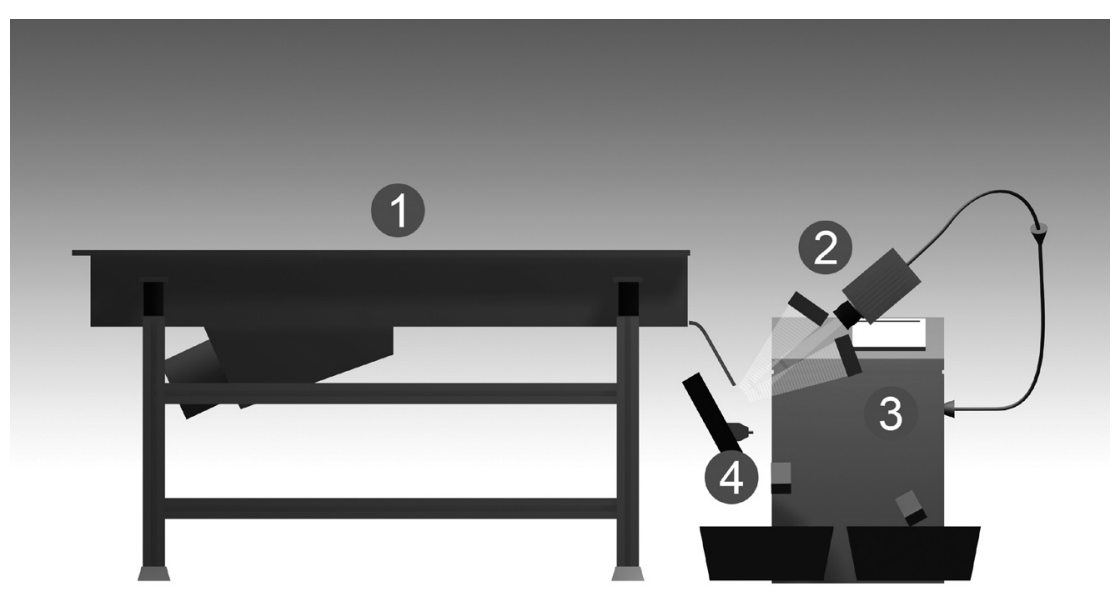

1. Vereinzelung 3. Klassifizierung

2. Erkennung 4. Trennung

Abb. 1: Prinzipskizze Rinnensortierer

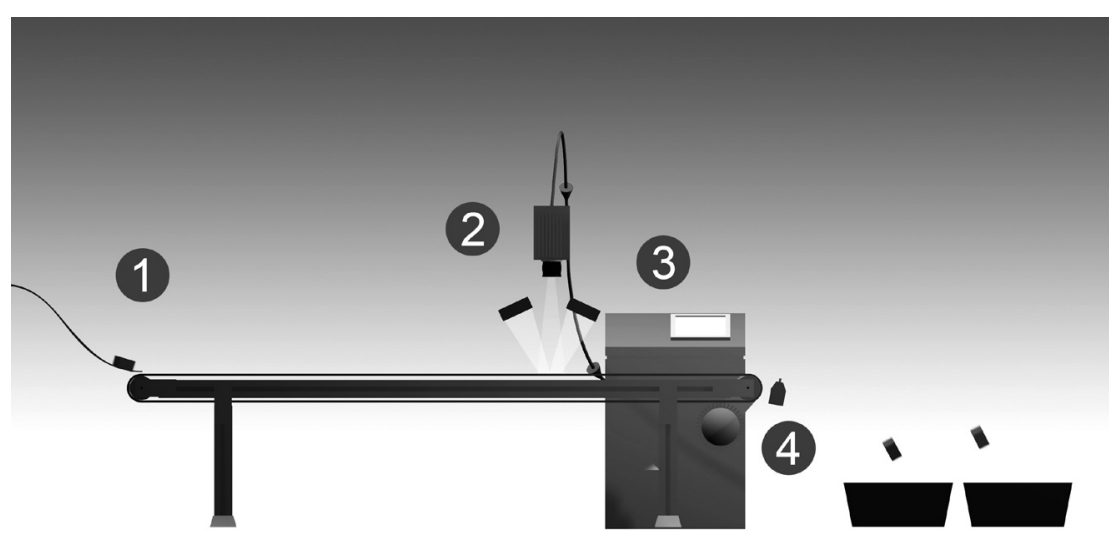

1. Vereinzelung 3 3lassifizierung

2. Erkennung 4. Trennung

Abb. 2: Prinzipskizze Bandsortierer

teile klassifiziert wird. Innerhalb von Millisekunden werden dann positionsgenau Druckluftventile in der Düsenleiste (4) aktiviert, so dass positiv detektierte Komponenten hinter einem Trennscheitel selektiv ausgetragen werden. Die anwenderseitig programmierbare Software des Rechners gestattet, dass die Klassifizierung in unterschiedliche Farben in einem sehr weiten Spektrum eingestellt werden kann. Dabei genügen auch geringe Farboder Helligkeitsunterschiede, die wahrnehmbar sind.

Da die meisten in Abfällen enthaltenen Stoffgruppen nicht die Eigenschaft „Schüttgut" erfüllen, wurden Bandmaschinen entwickelt, die insbesondere auch für grobstückiges und unregelmäßig geformtes Aufgabegut zur Anwendung kommen. Sortiermaschinen des Bandtyps sind nach dem in Abbildung 2 gezeigten Schema aufgebaut, wobei sich die nachstehende kurze Beschreibung auf eine Sortiermaschine mit Detek- mit dem menschlichen Auge kaum tion im Nahinfrarot- (NIR-) Wellenlängenbereich bezieht. Hierbei wird das zu trennende Material zunächst über eine Schwingförderrinne (in der Abbildung nicht gezeigt), eine geneigte Schurre (1) dem mit ca. 2,5 bis $3,0 \mathrm{~m} / \mathrm{s}$ umlaufenden Gurtförderer des Sortierers aufgegeben. Durch die kontinuierlich steigenden Fördergeschwindigkeiten wird eine Vereinzelung erzielt. Über dem Gurtförderer sind zwei mit Halogenlampen bestückte Lichtleisten sowie ein NIR-Sensor installiert (2), der die gesamte Gurtbreite abtastet und einem angeschlossenen Rechner (3) ein charakteristisches Spektrum der untersuchten Stoffe aufnimmt. Die Signale werden mit den in einer Datenbank gespeicherten Spektren verglichen. In Bruchteilen einer Sekunde erfolgt sodann die Klassifizierung, wobei auch die Lage und die Größe der Teile auf dem Gurt ermittelt werden. Die eigentliche Sortierung erfolgt mittels einer Luftdüsenleiste (4), die vor der Abwurftrommel des Gurtförderers angeordnet ist. Diese besteht aus vielen Einzeldüsen im Abstand von ca. 10-30 mm, die über Magnetventile angesteuert und aus einem Druckluftvorratsbehälter gespeist werden. Der Rechner gibt bei positiver Erkennung von auszutragenden Bestandteilen je nach Korngröße Signale an ein oder mehrere Ventile ab. Durch die Druckluftstöße werden die gewünschten Teile getrennt vom übrigen Stoffstrom ausgetragen. Die Erkennungsgenauigkeit, insbesondere eine präzise Identifikation von Korngröße und Kornform sowie der Lagebestimmung auf den Gurtförderer, kann durch den zusätzlichen Einbau einer Farbzeilenkamera als zweitem Sensor erheblich verbessert werden. Außerdem werden Sortiermaschinen hergestellt, die zwei Austragsdüsenleisten aufweisen. Somit lassen sich bei entsprechender Anordnung von zwei Trennscheiteln zwei Produkte in einem Arbeitsgang abtrennen. 


\section{Spektralbereiche}
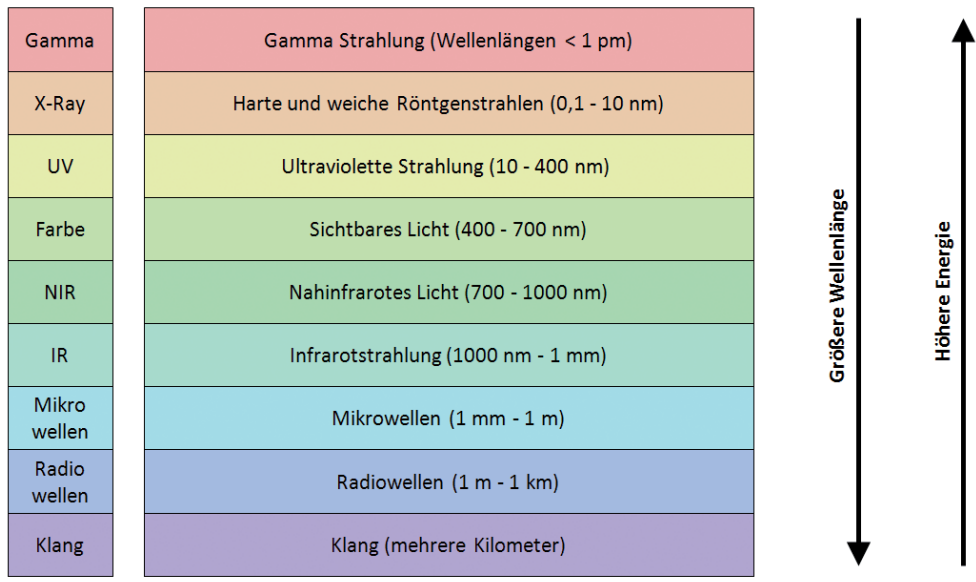

Abb. 3: Strahlungsarten für Emitter bei der sensorgestützten Sortierung

\subsection{Sensorsysteme}

Maschinen zur automatischen Klaubung enthalten neben der Materialzuführungs- und Vereinzelungseinheit sowie dem mechanischen Austrag eine Sensoreinheit. Die Komplexität der technischen Ausführung dieses Erkennungsmoduls ist maßgeblich entscheidend für die Einsatzmöglichkeiten der jeweiligen Sortiermaschinen. Grundsätzlich besteht bei der berührungslosen $\mathrm{Ab}$ tastung eines Fördergutstromes jede Sensoreinheit aus mindestens einem Emitter und einem Detektor. Der Emitter sendet elektromagnetische Strahlung in Richtung der zu untersuchenden Einzelstücke aus. Diese beeinflussen selektiv die Strahlung hinsichtlich des Spektrums, des zeitabhängigen Verlaufs, der Amplitude und der Richtung ihrer Ausbreitung. Die charakteristisch veränderte Strahlung trifft dann auf den Detektor und wird dort gemessen. Die Messdaten werden digital erfasst, im Rechner aufbereitet und soweit $\mathrm{zu}$ „Merkmalen“ reduziert, dass eine Sortierentscheidung getroffen werden kann. Somit kann direkt eine zeit- und positionsgenaue Ansteuerung der Austragseinheit erfolgen.

Bei der räumlichen Anordnung von Emitter und Detektor wird zwi- schen Reflektions- und Transmissionsmessung sowie angeregter Eigenemission unterschieden. Zur Messung von Reflexion sind Emitter und Detektor auf der gleichen Seite des Förderstroms angeordnet. Im sichtbaren Spektralbereich wird dieses Verfahren vor allem bei der Farbsortierung eingesetzt. Bei der Transmissionsmessung liegen Emitter und Detektor einander gegenüber. Mit dieser Anordnung lässt sich beispielsweise die Absorption von Röntgenstrahlung ermitteln. Darüber hinaus kann mit Strahlung hoher Energiedichte Materie zur Emission messbarer Eigenstrahlung angeregt werden. Dieses Verfahren der stimulierten Emission ermöglicht beispielsweise die Sortierung nach dem Merkmal der selektiven Fluoreszenz [Kill07].

\subsubsection{Spektralbereiche}

Die vom Emitter ausgesandte und im Detektor gemessene elektromagnetische Strahlung wird unter anderem durch ihre spektrale Zusammensetzung beschrieben. Je kürzer die Wellenlänge ist, desto höher ist die Energie der Strahlung. Bei der sensorgestützten Sortierung wird Strahlung in verschiedenen Spektralbereichen verwendet. Abbildung 3 zeigt die häufigsten Strahlungsarten zwischen Schallwellen und Gammastrahlung. Besonders häufig werden der sichtbare, der nahinfrarote und der Röntgen-Spektralbereich verwendet. Im sichtbaren Bereich finden Leuchtmittel wie Halogenlampen, Leuchtstoffröhren oder Leuchtdioden- (LED-) Leisten Verwendung als Emitter. Das von diesen ausgesendete „weiße“ Licht ist eine Mischung aus Farbtönen, die unterschiedlich stark von den Objektoberflächen absorbiert und reflektiert werden. Das vom Objekt reflektierte Licht wird zunächst spektral in Rot-, Grün- und Blauanteil zerlegt und anschließend mit CCD (Charge-coupled Device) oder CMOS (Complementary Metal Oxide Semiconductor) Sensoren erfasst.

Im nahen Infrarotbereich dienen überwiegend Halogenlampen als Emitter. Neben dem sichtbaren Spektrum senden diese auch einen großen Anteil an Infrarotstrahlung aus. Das von den zu messenden Abfallstücken reflektierte Spektrum wird in der Regel mit einem bewegten Spiegelsystem zunächst einem Spektrometer zugeführt und dort spektral zerlegt. Die Intensitätsverteilung bekannter Wellenlängen kann dann als Merkmal für unterschiedliche Stoffgruppen verwendet werden.

Metalldetektoren arbeiten meist im langwelligen Bereich der Radiofrequenzen. Das dabei von einer Erregerspule aufgebaute magnetische Wechselfeld wird durch die Objekte im Materialstrom beeinflusst. Eine Empfängerspule detektiert das resultierende Feld und vergleicht die Phasenlage und Amplitude mit den Parametern des Ursprungsfeldes. Hiermit können elektrisch leitfähige Stoffe wie Metalle von nicht leitfähigen Stoffen unterschieden werden, auch wenn sie nur in geringen Massenanteilen, wie etwa auf elektronischen Bauteilen vertreten sind.

\subsubsection{Sensorarten}

Die in sensorgestützten Sortiersystemen eingesetzten Detektoren un- 
terscheiden sich vor allem in ihrer Dimension und dem messbaren Spektralbereich. So werden Punkt-, Zeilen- und Flächensensoren unterschieden. Punktsensoren können zu einem Zeitpunkt nur eine Stelle des Förderstroms erfassen. Soll der Förderstrom jedoch lückenlos abgetastet werden, so muss der Messbereich über ein schnell arbeitendes Spiegelsystem geleitet werden. Die Informationsdichte in Form von Messpunkten pro Fläche ergibt sich aus der Taktrate des Punktsensors sowie der Geschwindigkeit und Präzision des Umlenksystems. Durch die schnell bewegten mechanischen Komponenten der Umlenkeinheit ergeben sich Grenzen für die Realisation räumlich hochauflösender Sensorsysteme. Soll beispielsweise ein mit $3 \mathrm{~m} / \mathrm{s}$ Fördergeschwindigkeit laufendes und $1.000 \mathrm{~mm}$ breites Band mit einer Auflösung von 1 pel/ mm (Messpunkt pro Millimeter) in und quer zur Förderrichtung abgetastet werden, so müssen pro Sekunde $3 * 10^{6}$ Punktmessungen durchgeführt werden. Die hieraus resultierende extrem kurze Sensorbelichtungszeit stellt eine weitere Grenze des Verfahrens zur lückenlosen Stoffstromerfassung dar. Bedingt durch die Umlenkmechanik hat jeder Messpunkt einen Versatz in Bandrichtung, welcher durch die Recheneinheit kompensiert werden muss. Außerdem stellt die Umlenkmechanik eine weitere Fehlerquelle dar. Punktsensoren werden daher hauptsächlich zur Unterstützung von Liniensensoren mit gezielt durchgeführten Scans kleinerer Abschnitte eingesetzt.

Liniensensoren scannen in Form eines Feldes von nebeneinander liegenden Sensorzellen den Stoffstrom zeilenweise $\mathrm{ab}$. Zu einem Zeitpunkt werden also die Messwerte für einen schmalen Abschnitt des Förderstroms gleichzeitig erfasst. Die Auflösung quer zur Förderrichtung ist dabei von der Bauform des Sensors abhängig. Soll der zuvor beschriebene Stoffstrom mit einer Auflö- sung von $1 \mathrm{~mm} /$ pel erfasst werden, so wird eine Sensorzeile mit 1.000 Sensorzellen benötigt. Das Bild des Stoffstroms wird dabei beispielsweise über eine Optik auf dem oft nur wenige Millimeter großen Sensor abgebildet. Zeilensensoren können jedoch auch direkt unter dem Stoffstrom in Dimensionen von mehreren Metern Breite unmittelbar zur Messung eingesetzt werden. Durch das parallele Scannen ergeben sich längere Belichtungszeiten der Sensorzellen im Vergleich zu Punktsensoren. Im obigen Beispiel genügen 3.000 Abtastungen pro Sekunde, um die gleiche Informationsdichte von $1 \mathrm{~mm} /$ pel zu erreichen, was einer Reduktion der Abtastrate um den Faktor 1.000 entspricht [Kill07].

Flächensensoren bestehen aus einer Matrix von Punktsensoren. Hierdurch können zum Zeitpunkt der Messung räumlich differenzierte Informationen in zwei Raumrichtungen erfasst werden. Aufgrund dieser Eigenschaft können sie bei der Abtastung von bewegten Stoffströmen dazu genutzt werden, höher dimensionale Informationen pro Stoffstromabschnitt und Messpunkt zu ermitteln als Zeilensensoren.

\subsubsection{Datenverarbeitung}

Selbst bei der Verwendung eines einzelnen Sensors fallen sehr große Datenmengen an, welche zur Ermittlung einer Sortierentscheidung in sehr kurzer Zeit präzise verarbeitet werden müssen. Dabei wird zwischen messpunktbasierter und objektbasierter Datenverarbeitung unterschieden. In jedem Fall wird jedoch aus dem mehrdimensionalen Datenstrom die Information soweit reduziert, dass die Austragseinheit eindeutig angesteuert werden kann. Die zur Verfügung stehende Bearbeitungszeit hängt dabei von der Bandgeschwindigkeit und dem Abstand zwischen Erkennungs- und Austragseinheit ab. Im oben betrachteten Beispiel eines mit $3 \mathrm{~m} / \mathrm{s}$ bewegten Förderstroms und einem Abstand von einem Meter zwischen Sensor und Austragseinheit stehen also ca. 300 ms für die Ausführung der gesamten Algorithmik zur Verfügung. Liegen Sensor und Austragseinheit, wie z. B. bei Rinnenmaschinen, nur wenige Zentimeter auseinander, so stehen nur wenige Millisekunden zur Entscheidungsfindung zur Verfügung.

Bei der messpunktbasierten Datenverarbeitung wird jeder Messpunkt direkt klassifiziert und einer Operation zur Steuerung der Austragseinheit zugeordnet. Wird beispielsweise ein Altglas-Stoffstrom mit einer Zeilenkamera im Transmissionsverfahren gescannt, so können lichtundurchlässige Bestandteile anhand der Helligkeit am Messpunkt identifiziert werden. An den entsprechenden Positionen wird dann zum richtigen Zeitpunkt ein Druckluftventil geöffnet und trägt die opaken Teile aus dem Stoffstrom aus. Dieses Verfahren arbeitet sehr schnell, allerdings können Form, Länge, Breite oder Helligkeitsverteilung von einem einzelnen Teilchen nicht ermittelt werden, da die einzelnen Messpunkte nicht in Bezug zueinander gesetzt, sondern isoliert voneinander verarbeitet werden müssen.

Bei der objektbasierten Datenverarbeitung werden die Messpunkte für einen Abschnitt des Förderbandes laufend in einem Puffer zwischengespeichert. In diesem Puffer befinden sich dabei üblicherweise mehre hundert Bildzeilen, in denen nach zusammenhängenden Punktmengen gesucht wird. Über die sogenannte Objekterkennung werden möglichst diejenigen Messpunkte zusammengefasst und in einen weiteren Puffer geschrieben, die zu einem Einzelstück auf dem Förderband gehören. Anschließend werden die Daten über Klassifikatoren $\mathrm{zu}$ einer Sortierentscheidung reduziert. Dabei können unter anderem auch Verhältnisse von Materialklassen pro Objektodergeometrische 


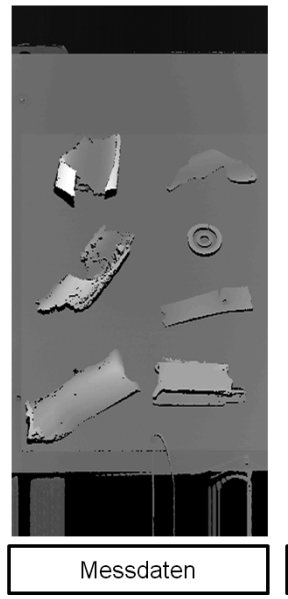

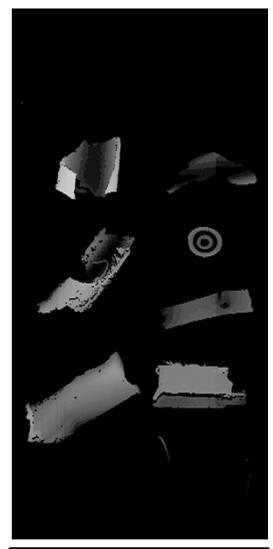

Band entfernt

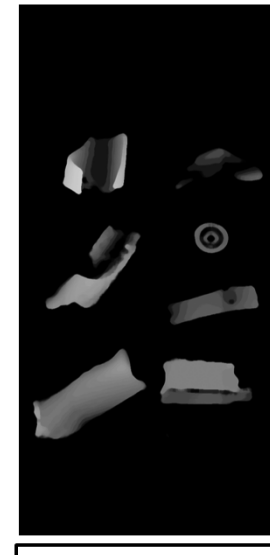

Störungen filtern

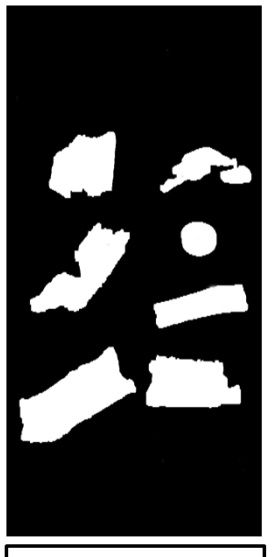

Flächen reduzieren
Abb. 4: Filterung eines Kamerabildes zur Identifizierung der Objektgrenzen

Daten wie Länge und Breite und, je nach Sensorart, auch das Volumen ermittelt werden. Zudem bietet die Objekterkennung die Möglichkeit der Zuordnung eines Einzelstücks in ein Koordinatensystem, was den Bezug zu nachfolgenden Sensordaten auch in Form von ergänzenden Punktmessungen ermöglicht.

Aufgabe der Datenverarbeitung ist neben der Klassifizierung der Messwerte und Extraktion von Merkmalen auch die Unterscheidung der Objekte vom Hintergrund. Der Hintergrund, also beispielsweise die Oberfläche eines Förderbandes, muss sich dabei in seinen Eigenschaften zur Strahlungsveränderung von dem zu sortierenden Material unterscheiden. Bei einer Farbsortierung bedeutet dies, dass sich die Bandoberfläche in Helligkeit oder Farbe von dem zu sortierenden Abfall deutlich unterscheiden muss. Dies stellt insbesondere dann eine große Herausforderung an die Robustheit der Technik dar, wenn sich durch Verschmutzungen die Bandfarbe der Objektfarbe anpasst [Kill07]. Abbildung 4 zeigt exemplarisch die schrittweise Filterung eines Kamerabildes zur Ermittlung der Objektgrenzen und damit der Abgrenzung von Objekt und Hintergrund. Im ersten Schritt wird ein Großteil des Förderbandes durch einen Schwellwertfilter entfernt. Anschließend werden Störungen durch ein Erosions- und Dilatationsfilter reduziert, um schließlich die Objektgrenzen eindeutig festlegen zu können. Durch Datenreduktion wird somit aus einem Sensorbild mit 8bit Informationstiefe ein 1bit-Bild erzeugt.

\section{Anwendungen}

Aktuelle Entwicklungen der letzten Jahre auf dem Gebiet der automatischen Klaubung belegen, dass die Palette der sortierfähigen Stoffe immer größer wird. Dies ist auf die ständige Weiterentwicklung neuer Detektoren und die zunehmende Implementierung von Sensorkombinationen zurückzuführen. Tabelle 1 vermittelt ohne Anspruch auf Vollständigkeit einen Überblick zu den detektierbaren, materialabhängigen Trennmerkmalen, den verwendeten Sensoren und typischen Anwendungen.

Zum Beleg der Leistungsfähigkeit und neuer Entwicklungen bei der automatischen Klaubung sind nachstehend exemplarisch einige Einsatzgebiete näher erläutert.

\subsection{Sortierung im sichtbaren Spektralbereich}

Die Sortierung von Abfallbestandteilen nach Farbe ist das älteste Anwendungsgebiet der sensorgestützten Sortierung, insbesondere für Altglas und zur Abscheidung nicht transparenter, glasfremder Inhaltsstoffe wie Keramik, Steine und Porzellan (KSP). Hierbei werden in der Regel Rinnenmaschinen eingesetzt, die als Sensoren eine oder zwei Farbzeilenkameras verwenden, die den Fördergutstrom von einer oder zwei Seiten inspizieren [Zei05]. Im Fall der selektiven Abtrennung von opaken KSP- Bestandteilen muss eine Transmissionsmessung erfolgen, während beispielsweise bei der Verarbeitung von Hohlglasscherben zur Gewinnung von sortenreinen Weiß-, Braun- und Grünglasprodukten auch eine Reflexionsmessung vor weißem Hintergrund möglich ist.

Weitere Einsatzmöglichkeiten der Farbsortierung bieten sich insbesondere beim Kunststoffrecycling an. Hier fallen häufig Mahlgüter im Korngrößenbereich von ca. 3 bis $12 \mathrm{~mm}$ an, die zumeist mit Rinnenmaschinen verarbeitet werden. Typisches Beispiel hierfür sind PETFlakes. Beim PET Recycling stellt die Faserindustrie den wichtigsten Absatzmarkt dar, wobei hohe Anforderungen an die Produktqualität eingehalten werden müssen. So darf der Anteil an Farbverunreinigungen im Recyclat nach einer erfolgreichen Farbsortierung nur noch maximal 100 ppm betragen [Zei04]. Weiterhin muss der Gehalt anderer Fremdstoffe im einstelligen ppm Bereich liegen. Bei einer Verwendung von sehr hochauflösenden CCD-Farbzeilenkameras lassen sich bereits Teilchen ab $2 \mathrm{~mm}$ Korngröße und dunkle Einschlüsse in den Kunststoffpartikeln ab 0,5 $\mathrm{mm}$ eindeutig identifizieren, so dass die Vorgaben an die Produktreinheit eingehalten werden können.

\subsection{Sortierung im infraroten Spektralbereich}

Sortiermaschinen, die das reflektierte, nicht sichtbare Licht im nahen Infrarotbereich (NIR) zur Erkennung nutzen, wurden zunächst erfolgreich für die Artikeltrennung 
Tab. 1: Typische Anwendungsgebiete der sensorgestützten Sortierung

\begin{tabular}{|c|c|c|}
\hline Trennkriterium & Sensorik & Sortierung von \\
\hline Farbe, Helligkeit & $\begin{array}{l}\text { Farbkameras und } \\
\text { VIS Spektrometer }\end{array}$ & $\begin{array}{l}\text { Altglas (Hohl- und Flachglas), Kunst- } \\
\text { stoffen, PET- Flakes, Granulaten, Kupfer } \\
\text { und Messing aus Nichteisenmetallge- } \\
\text { mischen, Platinen aus Elektronikschrott }\end{array}$ \\
\hline Transparenz & Farbkameras & opaken Bestandteilen aus Altglas \\
\hline Farbe, Glanz & Farbkameras & Trennung von Illustrierten aus Altpapier \\
\hline $\begin{array}{l}\text { molekulare Zusam- } \\
\text { mensetzung an der } \\
\text { Oberfläche }\end{array}$ & NIR- Spektrometer & $\begin{array}{l}\text { Kunststoffen aus LVP und anderen } \\
\text { Abfällen, Getränkekartons aus LVP, } \\
\text { Kunststoffgemischen in PE, PP, PS, PA, } \\
\text { PET, PVC, Papier, Pappe Kartonagen aus } \\
\text { Abfallgemischen, Holz aus Sperrmüll und } \\
\text { anderen Abfällen, Mischkunststoffen, } \\
\text { Papier, Holz und Textilien aus Abfallge- } \\
\text { mischen zur Ersatzbrennstoffherstellung, } \\
\text { PVC aus Abfallgemischen zur Chlorab- } \\
\text { reicherung }\end{array}$ \\
\hline $\begin{array}{l}\text { elektrische Leit- } \\
\text { fähigkeit }\end{array}$ & $\begin{array}{l}\text { induktive Detek- } \\
\text { toren }\end{array}$ & $\begin{array}{l}\text { Metallen aus diversen Abfallgemischen, } \\
\text { insbesondere aus Shredderrückständen, } \\
\text { Edelstählen aus Metallgemischen }\end{array}$ \\
\hline Dichte & $\begin{array}{l}\text { Röntgen- } \\
\text { detektoren }\end{array}$ & $\begin{array}{l}\text { Aluminium aus NE- Metallgemischen, } \\
\text { Elektronikkleingeräten aus LVP, Inert- } \\
\text { stoffen aus Abfallgemischen und Altholz, } \\
\text { PVC und Gummi aus Shredderrückstän- } \\
\text { den, organischen Verunreinigungen aus } \\
\text { Bauabfällen }\end{array}$ \\
\hline
\end{tabular}

aus Leichtverpackungsabfällen (LVP) eingesetzt. Heute ist ein sehr breiter Anwendungsbereich zur Wertstoffrückgewinnung u. a. in Sortieranlagen für die Verarbeitung von vorbehandelten Haushalts-, Gewerbe- und Baumischabfällen im Korngrößenbereich zwischen ca. 25 bis $400 \mathrm{~mm}$ gegeben. Grundsätzlich können mit NIR-Detektoren Getränkekartons, Papier, Pappe und Kartonagen (PPK), Holz, Windeln, Mischkunststoffe mit und ohne PVC sowie einzelne Kunststoffsorten wie PE, PP, PS, EPS, PA, PET und PVC selektiv identifiziert und abgeschieden werden. Die Güte des Trennerfolges wird allerdings oft durch das Vorhandensein schwarzer und sehr dunkel eingefärbte Materialien negativ beeinflusst, da hierbei das NIR-Licht so weitgehend absorbiert wird, dass keine reflektierte Strahlung die Sensorik erreicht [Hüs06]. Das Wertstoffausbringen beträgt deshalb in Abhängigkeit von der Beschaffenheit der Abfallgemische ca. 80 bis 90 \%. Die erzielbaren Pro- duktreinheiten erreichen 90 bis 97 Ma.-\%.

Ein neuerschlossenes, wichtiges Gebiet für die Sortierung im nahinfraroten Bereich ist die Herstellung von Ersatzbrennstoffen (EBS), die beispielsweise häufig in Anlagen zur Gewerbeabfallaufbereitung und in der mechanisch-biologischen Abfallbehandlung (MBA) erfolgt [Nis06]. Hier dienen NIR-Sortierer in der Regel zur Abtrennung hochkalorischer EBS-Bestandteile wie von Kunststoffen, PPK, Textilien und Holz, die sich mit konventionellen Verfahren wie der Windsichtung nur sehr unvollkommen erfassen lassen. Bei der Erkennung können störende Inhaltsstoffe wie und PET) ausgeblendet oder auch positiv aussortiert werden. Somit können Ersatzbrennstoffe deutlich von Schadstoffen abgereichert werden, wobei z. B. der Chlorgehalt auf unter $1 \%$ reduziert werden kann.

Eine innovative Entwicklung auf dem Sektor der Infrarottrennung Chlor- und Antimonträger (PVC betrifft die Sortierung von Altpapier. Hiermit kann mit gutem Trennerfolg sogenanntes Deinking-Material (Zeitschriften, Illustrierte, Prospekte) von Pappen und bedruckten Kartonagen separiert werden. Die für diesen Zweck bislang verwendete Sensortechnologie bestand aus einer Kombination aus Farbbildverarbeitung und NIR-Spektroskopie, die allerdings unzureichende Trennergebnisse erbrachte. So konnten bei einer Positivsortierung zwar braune Pappen gut erkannt werden, das Ausbringen an farbig bedruckten sowie grauen und weißen Kartonagen betrug jedoch nur etwa $50 \%$ bzw. $20 \%$. Außerdem wurde ein erheblicher Anteil an Deinkingware fehlausgetragen. Die Lösung ist eine neue Sortiermaschine des Bandtyps, über der ein Strahler mit einem hohen Anteil an mittelwelligem Infrarotlicht (MIR) installiert ist [Bou08]. Eine ebenfalls über dem Gurtförderer angeordnete Flächenkamera erfasst das reflektierte Spektrum, sowohl direkt unter dem Emitter als auch in nicht bestrahlten Bereichen, wobei die rechnergestützte Bildauswertung beide Zonen berücksichtigt. Dieses Messprinzip und die Anwendung geeigneter Auswertealgorithmen gestatten eine Erkennung des Flächengewichts der Komponenten des Aufgabematerials, so dass Pappe mit z. B. mehr als ca. $225 \mathrm{~g} / \mathrm{m}^{2}$ identifiziert und mittels Blasdüsen selektiv ausgetragen werden kann. Da im MIR- Bereich praktisch alle organischen Stoffe ca. 95 \% der auftreffenden Strahlung absorbieren, hat die Farbe der Oberfläche keinen Einfluss auf das Messergebnis. Somit können sogar schwarze Pappen und Kartonagen sowie auch dickwandigere papierfremde Bestandteile wie Kunststoffe und Metalle in einem Arbeitsgang identifiziert und ausgeschleust werden. Bei einem industriellen Einsatz einer derartigen Sortiermaschine konnte eine Reinheit des Deinkingmaterials von ca. 97 Ma.-\% bei einem Fehlaus- 
bringen von 14 bis $15 \%$ erzielt werden [Bou08].

\subsection{Metallsortierung mittels induktiver Detektoren}

Der rasante Preisanstieg für Altmetalle in den letzten Jahren hat die Verbreitung von Sortiermaschinen mit induktiver Sensorik deutlich gefördert. Diese Sortierer nutzen das Trennkriterium unterschiedlicher elektrische Leitfähigkeiten. Sowohl Band- wie auch Rinnenmaschinen können mit derartigen Sensoren ausgerüstet werden. Wegen der schlechten Schüttguteigenschaften der meisten Schrottarten werden jedoch zumeist Scheider des Bandtyps eingesetzt. Hierbei befindet sich die Sensorik in Form eines Spulensystems im Untertrum eines Bandförderers. Da die magnetische Induktion linear im Quadrat der Entfernung von den Spulen abnimmt, müssen die Spulen möglichst dicht unter dem Gurt angeordnet sein. Dabei sind Sensoren zur Erkennung aller Metalle wie auch zur Identifikation von Edelstählen einsetzbar. Bei Einführung dieser Technologie waren Spulen mit Durchmessern von $25 \mathrm{~mm}$ der übliche Standard. Heute stehen Detektoren mit 12,5 mm Arbeitsbreite zur Verfügung, wodurch sich die Ortsauflösung erheblich verbessert hat [Koh07]. Der zusätzliche Einbau einer Farbzeilenkamera ergibt den Vorteil einer Verbesserung der Bestimmung von Lage, Größe und Form der zu trennenden Bestandteile [Sch07]. Außerdem können hiermit auch Metallge- mische nach Farbe sortiert werden. So kann beispielsweise beim Elektronikschrottrecycling eine selektive Trennung von Nichteisenmetallen in graue Metalle (Aluminium, Zink, Blei) und rot/gelbe Metalle in Form von Kupfer und Messing erfolgen.

Ein Haupteinsatzgebiet induktiver Sortiersysteme liegt in der Verarbeitung von Shredder-Rückständen nach einer Wirbelstromscheidung. Hier gelingt es, noch enthaltene Nichteisen-Metalle und insbesondere Edelstahl weitgehend zurückzugewinnen, wobei insbesondere die selektive Abtrennung von Edelstählen von großem Vorteil ist, die vor der Verfügbarkeit induktiver Sortiersysteme nur unvollständig durch Handklaubung separiert werden konnten. Weiterhin gibt es eine Vielzahl von metallhaltigen Abfallgemischen, die sich vorteilhaft mit induktiver Sensorsortierung aufbereiten lassen.

\subsection{Röntgensortierung}

Eine Neuentwicklung der letzten Jahre stellen automatische Klaubemaschinen dar, die nach der Methode der Röntgentransmissions-Messung arbeiten. Dabei wird als Emitter eine Röntgenröhre verwendet, die über einem schnelllaufenden Gurtförderer angeordnet ist. Die Strahlung passiert die Komponenten des Aufgabegutes sowie auch den Gurt. Als Detektoren dienen zwei im Banduntertrum angeordnete, bildgebende Zeilensensoren [Har06]. Diese ermitteln die Intensität der auftreffenden Röntgenstrahlung gleichzeitig mit zwei Messkanälen in unterschiedlichen Wellenlängenbereichen. Die Auswertung der Messwerte beider Kanäle mittels Bildverarbeitung und geeigneter Algorithmen ergibt als Ergebnis eine Bestimmung der atomaren Dichte der Komponenten des Aufgabematerials. Dabei kann der Einfluss der Dicke der Einzelstücke (flächig oder körperförmig) auf die Strahlungsabsorption rechnerisch weitgehend aus dem Messresultat eliminiert werden.

Die Nutzung des Trennmerkmals stoffliche Dichte gestattet immer dann Anwendungen, wenn sich einzelne Komponenten in Abfallgemischen in ihrem spezifischen Gewicht unterscheiden. Dabei ist vorteilhaft, dass mit der Röntgenmethode eine trockene Sortierung ermöglicht wird. In der Metallaufbereitung können somit beispielsweise Magnesium und Aluminium von Schwermetallen getrennt werden. Weitere typische Applikationen sind die Erfassung von Elektronikschrott in Leichtverpackungs-Abfällen sowie die Separierung von Inertstoffen aus Baustellenabfällen und Altholz. Bei der Aufbereitung der Shredderleichtfraktion ist es mit der sensorischen Röntgensortierung gelungen, PVC, Elastomere und Metalle fast vollständig abzuscheiden [Jul07].

\section{Korrespondenzadresse:}

Prof. Dr.- Ing. Thomas Pretz

Dr.- Ing. Jörg Julius

Institut für Aufbereitung und Recycling

der RWTH Aachen

Wüllnerstraße 2

52062 Aachen

\section{LITERATUR}

[Bou08] MIR (4.-6. März, 2008) Technologie für die Papiersortierung, Vortrag zur Fachtagung Sensorgestützte Sortierung, Aachen

[Fea08] Fears P (4.-6. März, 2008) Maximizing Mechanical Metal Recovery, Eriez ProSort Mechanical Metal Sorter, Vortrag zur Fachtagung Sensorgestützte Metal Sorter, Vortrag $z$

[Har06] Harbeck H (28.-30. März, 2006) Aufbe[Har06] Harbeck H (28.-30. März, 2006) AufbeSortierung, Vortrag zur Fachtagung Sensorgestütze Sortierung, Vortrag
Sortierung, Aachen

[Hüs06] Hüskens J (2006) Sortenreine Wertstoffe
Sortierung, Aachen mit NIR - Nahinfrarot-Technik in Anwendungen bei der

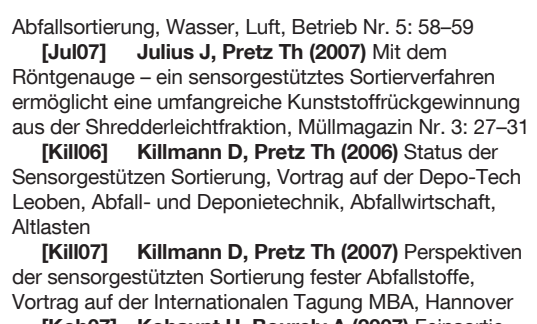
Vortrag auf der Internationalen Tagung MBA, Hannover
[Koh07] Kohaupt U, Bourely A (2007) Feinsortie-

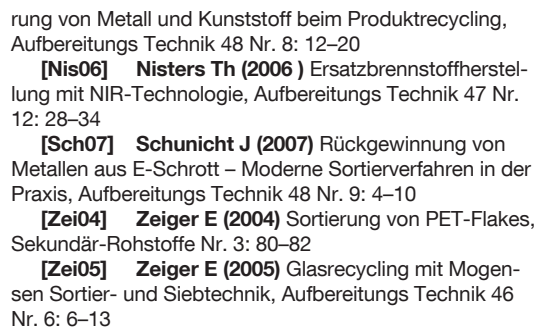

